\title{
Serum Calprotectin and Prealbumin Levels among Ovarian Cancer Patients Aged 30 - 45 Years
}

\author{
Anastasia Petsa ${ }^{1}$, Vasilios Pergialiotis ${ }^{1}$, Panagiotis Konstantopoulos ${ }^{1}$, Athanasia Katsichti ${ }^{1}$, \\ Kyriakos Petrou ${ }^{1}$, Athanasios Giannopoulos' ${ }^{2}$, Dimitrios Botsis ${ }^{3}$, Despina Perrea1 \\ ${ }^{1}$ Laboratory of Experimental Surgery and Surgical Research N.S. Christeas, National and Kapodistrian University of Athens, \\ Athens, Greece \\ ${ }^{2} 1^{\text {st }}$ Department of General Surgery, "Laikon" General Hospital of Athens, Athens, Greece \\ ${ }^{3} 2^{\text {nd }}$ Department of Obstetrics-Gynecology, “Aretaieion” Hospital, Athens, Greece \\ Email: *anapetsa@yahoo.gr
}

How to cite this paper: Petsa, A., Pergialiotis, V., Konstantopoulos, P., et al. (2017) Serum Calprotectin and Prealbumin Levels among Ovarian Cancer Patients Aged 30 45 Years. Open Journal of Obstetrics and Gynecology, 7, 303-311.

https://doi.org/10.4236/ojog.2017.73032

Received: February 9, 2017

Accepted: March 19, 2017

Published: March 22, 2017

Copyright $\odot 2017$ by authors and Scientific Research Publishing Inc. This work is licensed under the Creative Commons Attribution International License (CC BY 4.0).

http://creativecommons.org/licenses/by/4.0/

\begin{abstract}
Purpose: Serum calprotectin and prealbumin have been previously linked with ovarian cancer. However, to date, their effectiveness in the diagnosis of the disease remains uncertain. The purpose of the present study is to determine whether serum prealbumin and calprotectin correlate with the stage and type of histologic diagnosis. Materials-Methods: In the present study, we included 80 women aged 30 - 45 years that had either primary ovarian cancer (Stages 1 and 2) with elevated levels of CA-125 $(n=40)$ or were healthy $(n=$ 40). Serum calprotectin and prealbumin levels were assessed using ELISA kits. Statistical analysis was performed with SPSS v.20.0 statistical software. Results: Both plasma prealbumin and calprotectin levels were significantly different in patients with ovarian cancer compared to healthy controls. Specifically, serum prealbumin levels showed a level towards decreasing as the stage of the disease advanced (21.2 (15.5 - 30.6) in healthy controls, $12.8(6.9-16)$ for patients with stage 1 disease and $9.9(4.0$ - 15.5) for patients with stage 2 disease $p<0.001)$. Its sensitivity was $100 \%$ and specificity $82.5 \%$. Serum calprotectin, on the other hand, had an increasing motif (550 (100 - 2780) for healthy controls, 3320 (2580 - 7930) for patients with stage 1 disease and 3825 (2350 - 5990) for stage 2 disease $p<0.001$ ). Similarly, its sensitivity was $100 \%$ and specificity $77.5 \%$. Conclusions: Both prealbumin and calprotectin seem to be useful markers for the identification of patients suffering from ovarian cancer.
\end{abstract}

\section{Keywords}

CA-125, Calprotectin, Ovarian Cancer, Prealbumin 


\section{Introduction}

Ovarian cancer is considered the most lethal gynecological cancer in developed countries. It represents $4 \%$ of all new cancer cases in the United States of America (USA) and 25\% of all gynecological cancer cases. Furthermore, it is responsible for $5 \%$ of cancer-related deaths in women and $50 \%$ of deaths from gynecological cancers [1] [2] [3] [4]. Despite the significant progress made in the diagnostic approach and treatment of the disease, its mortality remains very high. This is attributed to the fact that $75 \%$ of the diagnosed cases concern advanced stages of disease [5] [6].

Several methods have been used in the general population with arbitrary efficacy for early diagnosis of the disease. Transvaginal sonography and serum cancer antigen 125 (CA-125) levels represent the most common implemented tests for the diagnosis and follow-up of the disease. They have been studied extensively-both separately as well as in combination [5] [6]. Nevertheless, none of them fully fulfil the criteria to be used as a screening test for the early diagnosis of ovarian cancer, even when they are applied in high-risk groups [2].

Calprotectin, also known as MRP-8/MRP-14 or S100A8/A9 heterocomplex, is formed out of the calcium-binding, migration inhibitory factor-related proteins, MRP-8 (myeloid-related protein-8 or S100A8) and MRP-14 (myeloid-related protein-14 and S100A9) [7] [8] [9]. The expression of these proteins is largely confined to the cytosol of neutrophils and monocytes. It comprises $60 \%$ of the cytoplasmic protein fraction of circulating polymorphonuclear granulocytes and is also found in monocytes, macrophages and ileal tissue eosinophils. Calprotectin has antibacterial, antifungal and immunomodulating and antiproliferative effects. Normal human plasma contains a calprotectin concentration ranging from 500 to $3.000 \mathrm{ng} / \mathrm{ml}$ [9]. Calprotectin is not only involved in promoting the inflammatory response in infections, but was also identified as a potent amplifier of inflammation in autoimmunity as well as in cancer development and tumor spread [7].

Prealbumin is a protein produced primarily by the liver [10]. It is also produced by the choroid plexus, by pancreatic islet cells in the embryonic yolk sac, and by enterochromaffin cells in the gastrointestinal mucosa [11]. It serves as a source of amino acids for the production of other proteins, and it carries substances such as the thyroid hormone thyroxine throughout the body. Prealbumin (PAB), also known as thyroxine binding prealbumin or transthyretin, is a stable circulating glycoprotein [10]. It is the preferred marker for protein malnutrition, since it is easily quantified, less affected by liver disease than other serum proteins, and has a half-life of only two days [11] [12]. Its levels vary by age. Levels lower than $11 \mathrm{mg} / \mathrm{dL}$ indicate severe nutritional deficiency. Normal levels of prealbumin for adults range from 15 to $36 \mathrm{mg} / \mathrm{L}$ [10] [13].

There seem to be contradictive reports regarding the levels of pre-operative plasma calprotectin in patients with ovarian cancer [14] [15]. Serum pre-albumin levels have also been studied preoperatively as a nutritional assessment tool which could predict the patients' response to primary radical cytoreductive sur- 
gery [10]. However, to date we do not know actually whether calprotectin might also be useful as a screening tool for ovarian cancer diagnosis.

The purpose of the present study is to determine the efficacy of serum prealbumin and calprotectin in detecting patients with ovarian cancer.

\section{Materials and Methods}

\subsection{Patients}

The present study was conducted in a consecutive sample of women with primary ovarian cancer that attended the gynaecological clinics of "Laikon" and "Aretaieion" Hospitals in Athens, Greece, as well as the gynecological clinics of "Metaxa" and "Tzaneion" Hospitals in Piraeus, Greece. The study was approved by the Institution Ethics Committee of all hospitals. All examinations were in accordance with the ethical standards of the institutional and/or national research committee and with the 1964 Helsinki declaration and its later amendments or comparable ethical standards. The patient's age ranged between 30 and 45 years. This prospective cohort study took place between December 2012 and January 2015. It was a prospective observational study. No randomization took place; hence, the sample size was not pre-determined.

We stratified these women in two distinct groups. The first group (group A) consisted of women with primary ovarian cancer and elevated CA-125 $(>500)$ $(\mathrm{N}=40)$. In the second group (group $\mathrm{B}$ ), we included 40 healthy women without any history of clinical and laboratory data of ovarian cancer. Both healthy women and patients, participating in the study, had a blood examination preoperatively, after giving a written consent. The questionnaire was designed and completed by the researchers, in order to assess the inclusion and exclusion criteria described in our study and presented in Table 1. It contained basic demographic information (such as age, weight, height), as well as information from their medical history (such as subfertility, the use of talk for cosmetic reasons, preterm menopause), family history (of ovarian or breast cancer, hereditary syndromes), current medical treatment for any other synchronous diseases (such as NSAIDs, corticosteroids, hormone replacement therapy, oral contraceptive pills, fertility treatment) and dietary habits (whether their diet was rich in proteins or fat).

\subsection{Inclusion Criteria}

The inclusion criteria in our study were the age range of 30 - 45 years old, the histological confirmation for primary ovarian cancer and the elevated levels of cancer antigen CA-125.

\subsection{Exclusion Criteria}

Patients were excluded if they were previously treated for ovarian cancer or any other form of cancer, if they used non-steroidal anti-inflammatory drugs (NSAIDS) and/or cortisone systematically, if they were under treatment with oral contraceptives or any other form of hormone replacement therapy, if they 
Table 1. Statistical analysis of differences among patients with epithelial ovarian cancer and healthy controls.

\begin{tabular}{ccccc}
\hline & Controls & Stage 1 & Stage 2 & $p$-value \\
\hline Age & $35(31-44)$ & $39.5(30-44)$ & $42(35-44)$ & 0.001 \\
Weight & $74(51-90)$ & $51(44-58)$ & $51.5(46-61)$ & $<0.001$ \\
BMI & $26(21-30)$ & $18(18-22)$ & $18(17-20)$ & $<0.001$ \\
Preterm menopause & $4 / 40$ & $12 / 20$ & $15 / 20$ & $<0.001$ \\
Talk use & $3 / 40$ & $20 / 20$ & $18 / 20$ & $<0.001$ \\
Subfertility & $2 / 40$ & $14 / 20$ & $17 / 20$ & $<0.001$ \\
Fertility treatment & $2 / 40$ & $13 / 20$ & $14 / 20$ & $<0.001$ \\
Ovarian or breast cancer & $5 / 40$ & $17 / 20$ & $16 / 20$ & $<0.001$ \\
Familial cancer & $0 / 40$ & $3 / 20$ & $4 / 20$ & $<0.007$ \\
CA-125 & $20.5(15-34)$ & $70.5(39-95)$ & $125.5(99-148)$ & $<0.001$ \\
Prealbumin & $21.2(15.5-30.6)$ & $12.8(6.9-16.0)$ & $9.9(4-15.5)$ & $<0.001$ \\
Calprotectin & $550(100-2780)$ & $3320(2580-7930)$ & $3825(2350-5990)$ & $<0.001$ \\
Abnormal prealbumin & $0 / 40$ & $15 / 20$ & $18 / 20$ & $<0.001$ \\
Abnormal calprotectin & $0 / 40$ & $16 / 20$ & $17 / 20$ & $<0.001$ \\
\hline
\end{tabular}

received amiodarone, or if they were pregnant, if they suffered from chronic renal failure, reported alcohol abuse, hyperthyroidism, Hodgkin's disease, hepatobiliary disease, nephrotic syndrome, systematic use of anabolics or androgens, and if they had current autoimmune diseases and/or infection (active or recent).

\subsection{Immunohistochemistry}

Blood was collected in Vacutainer tubes (BD Diagnostics, NJ, USA). Centrifugation was applied at $3000 \mathrm{rpm}$ (revolutions per minute) for 15 minutes. Then, from each serum sample, $200 \mu \mathrm{l}$ was placed in 5 Eppendorf and the specimens were stored at $-30^{\circ} \mathrm{C}$ until the assay (within 1 month). All measurements were performed in an ELISA photometer (Model 680 Microplate Reader; Bio-Rad ${ }^{\mathrm{TM}}$ ) at the Laboratory of Experimental Surgery and Surgical Research "N.S. Christeas". Serum calprotectin and prealbumin levels were assessed using ELISA kits provided by Cusabio Biotech Co. LTD.

\subsection{Statistical Analysis}

Statistical analysis was performed with SPSS v.20.0 statistical software (IBM Corp. Released 2011. IBM SPSS Statistics for Windows, Version 20.0. Armonk, NY: IBM Corp). The normality of the distributions was assessed with Kolmogorov-Smirnov's test and graphical methods. Data are expressed as median (range) for continuous data. Comparisons were performed with the non-parametric 
Kruskal-Wallis test for multiple testing. Categorical variables were analyzed with the two-tailed chi-square test. Linear regression was performed to study the correlation of serum CA-125 (independent variable) with both serum prealbumin and calprotectin (dependent variables). The Enter method was preferred. The level of statistical significance was set at $p<0.05$. The sensitivity and specificity of the method were assessed with the use of ROC curves.

\section{Results}

Overall, 80 women were included in the present prospective cohort study (Table 1). These were divided into two groups: 1) 40 women with primary ovarian cancer and with high levels of CA-125 in the blood who were further divided in two sub-groups, 20 (25\%) were stage 1 and 20 (25\%) were stage 2 (group A) and 2) 40 healthy women without any history of clinical and laboratory data of ovarian cancer (control group-group B).

The patients' age ranged between 30 and 45 years old. Among them 28.7\% $(\mathrm{N}=23)$ were 30 to 35 years old, $27.5 \%(\mathrm{~N}=22) 35$ to 40 years old and $43.8 \%$ $(\mathrm{N}=35) 40$ to 45 years old.

Prealbumin was low (less than $15 \mathrm{mg} / \mathrm{L})$ in $41.25 \%(\mathrm{~N}=33)$ of women and normal (from 15 to $30 \mathrm{mg} / \mathrm{L}$ ) in $58.75 \%(\mathrm{~N}=47)$. All women in the control group had normal levels of prealbumin (15.5 - $30.6 \mathrm{mg} / \mathrm{L})$. In group A, 15 patients with stage 1 and 18 patients with stage 2 disease had abnormal levels of prealbumin. The sensitivity of the method in detecting patients with ovarian cancer was $100 \%$ and its specificity $82.5 \%$. A significant correlation of serum prealbumin and CA-125 levels was observed $(\mathrm{r}=0.78, p<0.001)$.

As far as calprotectin is concerned, it was found elevated (more than 3000 $\mathrm{ng} / \mathrm{ml})$ in $42.5 \%(\mathrm{~N}=34)$ of women and within normal levels (from 500 to $3000 \mathrm{ng} / \mathrm{ml})$ in $57.5 \%(\mathrm{~N}=46)$. It was remarkable that all women in the control group had normal levels of calprotectin $(100-2780 \mathrm{ng} / \mathrm{ml})$ and most women with ovarian cancer had elevated levels; particularly those with stage 2 . More specifically, in sub-group stage 1, 16 out of 20 participants had elevated levels of calprotectin, while in sub-group stage 2, 17 out of 20 participants had elevated levels of calprotectin. Its sensitivity in detecting patients with ovarian cancer was also high (100\%) and its specificity $77.5 \%$. A significant correlation of serum calprotectin and CA-125 levels was observed $(r=0.67, p<0.001)$.

From their medical history, it was found that the use of talk in the vagina for cosmetic reasons, which is a known risk factor for ovarian cancer, was very high in Group A, since all women in stage $1(\mathrm{~N}=20)$ and almost all women in stage $2(\mathrm{~N}=18 / 20)$ referred using it in the past $(p<0.001)$. Moreover, women in Group A experienced preterm menopause or subfertility and had been submitted to fertility treatment $(\mathrm{N}=37, p<0.001)$. From their family history, the history of ovarian or breast cancer was present in $82.5 \%(\mathrm{~N}=33)$ of women in Group A. $(p<0.001)$ Nevertheless, the incidence of hereditary syndromes in the same group was very low, only in $17.5 \%$ of women in Group A $(\mathrm{N}=7, p<$ 0.007) (Table 1). 


\section{Discussion}

Our research has shown that the serum levels of calprotectin were elevated pre-operatively in the serum of women with ovarian cancer, compared to the control group. More specifically, the levels were significantly higher among patients with stage 2 disease. We have also observed that the levels of pre-albumin were respectively decreased in the group of women with ovarian cancer. Furthermore, from the use of our questionnaire, we have noticed a positive correlation of patients with ovarian cancer with most of the known risk factors, including preterm menopause, talk use, subfertility, fertility treatment, and family history of ovarian or breast cancer [3] [5].

In a study in Norway, researchers analysed calprotectin serum levels in women with ovarian carcinomas, borderline ovarian tumours (BOT), and benign ovarian tumours [16]. Serum Ca-125 was analysed in the same study population. They concluded that median plasma calprotectin concentration was elevated in ovarian carcinoma, compared with controls, as well as with BOT, but remarked that its effectiveness as a tumour-marker was inferior to that of CA-125. A positive correlation was also found between CA-125 and calprotectin concentrations in ovarian carcinoma. However, the exclusion criteria that they used were not as strict as those used in our study. Since calprotectin is considered a marker of intestinal inflammation, in order to have more reliable results, patients with intestinal diseases, such as inflammatory bowel disease (IBD), Chrohn's disease, ulcerative colitis, colon cancer, and treatment with non-steroidal anti-inflammatory drugs (NSAIDs), should be excluded [8] [17].

In another study in Norway, researchers analysed circulating endoglin concentration in ovarian carcinoma and evaluated the prognostic role for calprotectin and endoglin in advanced-stage disease [15]. More specifically, they compared preoperative plasma concentration of endoglin from women with benign ovarian tumours, borderline ovarian tumours, and ovarian carcinomas to endoglin and calprotectin concentrations in advanced-stage ovarian carcinoma. They concluded that endoglin and calprotectin concentrations did not correlate with survival, and that circulating endoglin is not elevated in advanced ovarian carcinoma.

As far as pre-albumin is concerned, there are two studies involving this potential biomarker. The first study, conducted in England, does not include it directly, but indirectly. Based on the observation that the presence of an ongoing systemic inflammatory response is associated with poor outcome in patients with advanced cancer (stage 3/4), they analysed C-reactive protein, and albumin levels in these women. They concluded that the presence of a systemic inflammatory response is an independent predictor of poor overall survival in patients with advanced ovarian cancer, independent of treatment received [18]. The drawback of this research was the use of albumin and not pre-albumin since albumin's short half-life is approximatively 21 days, making it difficult to monitor changes affecting the synthesis and catabolism of proteins [7] [13]. Moreover, its levels are affected by liver disorders, dehydration, or vitamin deficiency. That is 
why pre-albumin started being investigated in more studies, since its short half-life is approximately 2 days, which means that it breaks down quickly in the body and the amount changes rapidly, decreasing when there is a protein deficiency [11] [13]. In addition to this, our research included only women with stages 1 and 2 ovarian cancer.

The second study for pre-albumin was conducted in the U.S.A. and its purpose was to determine if serum pre-albumin could be used to objectively determine which patients could safely undergo cytoreductive surgery [10]. Patients with suspected ovarian cancer, in a 24-month period, underwent nutritional assessment during their preoperative workup and were followed for development of postoperative complications. They observed that postoperative complications increased with lower pre-albumin levels. All complications occurred in group of patients with pre-albumin $<18 \mathrm{mg} / \mathrm{dl}$. A significantly increased number of complications occurred in patients with pre-albumin $<10 \mathrm{mg} / \mathrm{dl}$. All postoperative mortality in this series occurred in patients with pre-albumin $<10 \mathrm{mg} / \mathrm{dl}$. Patients whose pre-albumin started low but was able to be raised to $>10 \mathrm{mg} / \mathrm{dl}$ did not have significantly increased complications compared to patients whose initial pre-albumin was $>10 \mathrm{mg} / \mathrm{dl}$. They concluded that significantly more blood loss, morbidity, and mortality occurred in patients with abnormal preoperative pre-albumin. This was especially true in patients with a pre-albumin $<10 \mathrm{mg} / \mathrm{dl}$. With these significantly increased risks, patients with extremely poor nutritional status (pre-albumin $<10 \mathrm{mg} / \mathrm{dl}$ ) may be better served by neoadjuvant chemotherapy with interval cytoreductive surgery if nutrition improves. The advantage of our research is the fact that we determine objectively the levels of pre-albumin. We managed to exclude conditions that can increase concentrations of pre-albumin, such as nephrotic syndrome, Hodgkin's disease, chronic renal failure, pregnancy, alcohol abuse, hyperactive adrenal glands, and medications (i.e. corticosteroids, progesterone and related agents, anabolic steroids, androgens and non-steroidal anti-inflammatory drugs [5] [10] [13]), as well as conditions that can decrease concentrations of pre-albumin, such as malnutrition, serious infections, burns, poisoning from salicylates, severe or chronic conditions such as cancer, hepatobiliary disease, hyperthyroidism, and, medications (i.e. oestrogen, contraceptive pills, and amiodarone [5] [10] [11] [16]).

\section{Strengths and Weaknesses of Our Study}

The main strength of our study is that the population included were women aged from 30 to 45 years, where ovarian cancer is rare, but more severe since it concerns young population. Another strength of our research is that we interviewed and obtained blood sample from all women who were suspect for ovarian cancer, meaning women with findings on the ovaries (either by ultrasound or by computed tomography-CT or magnetic resonance imaging-MRI), and only women with histologically confirmed ovarian cancer remained in our sample, while the rest (benign ovarian tumours, metastatic tumours or BOTs) were excluded. Moreover, exclusion criteria were much stricter compared to any other 
research done in this field, since we have detected every condition referred that can affect (decrease or increase) the levels of these two biomarkers and excluded them. We were, also, able to identify a positive correlation between both serum prealbumin and calprotectin with serum CA-125.

The weaknesses of our study are first of all the sample-size, which is attributed to the low incidence of ovarian cancer in this particular age group. Another drawback of our research is that we did not monitor these two biomarkers post-operatively and thus correlate them with survival rate.

\section{Implications for Current Clinical Practice in the Field and Future Research}

Further research is needed in this field to further clarify the potential use of these two biomarkers. Future studies should include older women (more than 65 years old) where ovarian cancer is more common, to define whether calprotectin and pre-albumin can be used as screening tools. Moreover, these markers can be monitored post-operatively in order to examine whether surgical removal results in decreases of their levels.

Concluding ovarian carcinoma has in general a poor prognosis since the vast majority of tumours are genomically unstable and clinically highly aggressive, resulting in rapid progression of malignancy potential while still asymptomatic, resulting in a late diagnosis. It is therefore of critical importance to develop methods to diagnose ovarian carcinoma at its earliest developmental stage in order to differentiate between benign tissue and their early malignant transformed counterparts [19].

According to the findings of our study, preoperative plasma prealbumin and calprotectin seem to be promising markers for the diagnosis of epithelial ovarian cancer.

\section{References}

[1] Centers for Disease and Control Prevention (2016) Ovarian Cancer Statistics. https://www.cdc.gov/cancer/ovarian/statistics/

[2] Barton, M.B. and Lin, K. (2012) Screening for Ovarian Cancer: Evidence Update for the U.S. Preventive Services Task Force Reaffirmation Recommendation Statement. Agency for Healthcare Research and Quality Publication No. 12-05165-EF3, Rockville.

[3] Moyer, V.A. (2012) Screening for Ovarian Cancer: U.S. Preventive Services Task Force Reaffirmation Recommendation Statement. Annals of Internal Medicine, 157, 900-904. https://doi.org/10.7326/0003-4819-157-11-201212040-00539

[4] Permuth-Wey, J. and Sellers, T.A. (2009) Epidemiology of Ovarian Cancer. Methods in Molecular Biology, 472, 413-437.

https://doi.org/10.1007/978-1-60327-492-0_20

[5] Argento, M., Hoffman, P. and Gauchez, A.S. (2008) Ovarian Cancer Detection and Treatment: Current Situation and Future Prospects. Anticancer Research, 28, 31353138.

[6] Doubeni, C.A., Doubeni, A.R. and Myers, A.E. (2016) Diagnosis and Management 
of Ovarian Cancer. American Family Physician, 93, 937-944.

[7] Ehrchen, J.M., Sunderkotter, C., Foell, D., Vogl, T. and Roth, J. (2009) The Endogenous Toll-Like Receptor 4 Agonist S100A8/S100A9 (Calprotectin) as Innate Amplifier of Infection, Autoimmunity, and Cancer. Journal of Leukocyte Biology, 86, 557-566. https://doi.org/10.1189/jlb.1008647

[8] Gebhardt, C., Nemeth, J., Angel, P. and Hess, J. (2006) S100A8 and S100A9 in Inflammation and Cancer. Biochemical Pharmacology, 72, 1622-1631.

[9] Gaya, D.R. and Mackenzie, J.F. (2002) Faecal Calprotectin: A Bright Future for Assessing Disease Activity in Crohn's disease. Oxford Journals, 95, 557-558.

[10] Geisler, J.P., Linnemeier, G.C., Thomas, A.J. and Manahan, K.J. (2007) Nutritional Assessment Using Prealbumin as an Objective Criterion to Determine Whom Should Not Undergo Primary Radical Cytoreductive Surgery for Ovarian Cancer. Gynecologic Oncology, 106, 128-131. https://doi.org/10.1016/j.ygyno.2007.03.008

[11] Beck, F.K. and Rosenthal, T.C. (2002) Prealbumin: A Marker for Nutritional Evaluation. American Family Physician, 65, 1575-1578.

[12] Spiekerman, A.M. (1995) Nutritional Assessment (Protein Nutriture). Analytical Chemistry, 67, R429-436. https://doi.org/10.1021/ac00108a026

[13] Cassels, C. (2016) Medscape Obstetrics/Gynaecology \& Women's Health: Prealbumin an Independent Predictor of Mortality/Morbidity.

http://www.medscape.com/viewarticle/538923

[14] Kostakis, I.D., Cholidou, K.G., Kallianidis, K., Perrea, D. and Antsaklis, A. (2010) The Role of Calprotectin in Obstetrics and Gynecology. European Journal of $\mathrm{Ob}$ stetrics and Gynaecology Reproductive Biology, 151, 3-9.

https://doi.org/10.1016/j.ejogrb.2010.03.006

[15] Odegaard, E., Davidson, B., Engh, V., Onsrud, M. and Staff, A.C. (2008) Assessment of Endoglin and Calprotectin as Potential Biomarkers in Ovarian Carcinoma and Borderline Tumors of the Ovary. American Journal of Obstetrics and Gynecology, 199, 533.e1-533.e8.

[16] Buys, S.S., Partridge, E., Black, A., et al. (2011) Effect of Screening on Ovarian Cancer Mortality: The Prostate, Lung, Colorectal and Ovarian (PLCO) Cancer Screening Randomized Controlled Trial. Journal of American Medical Association, 305, 2295-2303. https://doi.org/10.1001/jama.2011.766

[17] Ni Bhriain, H., Trovik, J., Wik, E., et al. (2009) Plasma Calprotectin Concentrations in Women with Endometrial Carcinoma. Gynecologic Oncology, 114, 491-495. https://doi.org/10.1016/j.ygyno.2009.06.008

[18] Sharma, R., Hook, J., Kumar, M. and Gabra, H. (2008) Evaluation of an Inflammation-Based Prognostic Score in Patients with Advanced Ovarian Cancer. European Journal of Cancer, 44, 251-256. https://doi.org/10.1016/j.ejca.2007.11.011

[19] Waldemarson, S., Krogh, M., Alaiya, A., et al. (2012) Protein Expression Changes in Ovarian Cancer during the Transition from Benign to Malignant. Journal of Proteome Research, 11, 2876-2889. https://doi.org/10.1021/pr201258q 
Submit or recommend next manuscript to SCIRP and we will provide best service for you:

Accepting pre-submission inquiries through Email, Facebook, LinkedIn, Twitter, etc. A wide selection of journals (inclusive of 9 subjects, more than 200 journals)

Providing 24-hour high-quality service

User-friendly online submission system

Fair and swift peer-review system

Efficient typesetting and proofreading procedure

Display of the result of downloads and visits, as well as the number of cited articles Maximum dissemination of your research work

Submit your manuscript at: http://papersubmission.scirp.org/

Or contact ojog@scirp.org 